\title{
INEQUALITIES AND BOUNDS FOR A CERTAIN BIVARIATE ELLIPTIC MEAN
}

\author{
EDWARD NEUMAN
}

Abstract. This paper deals with a new mean introduced recently by this author. This mean is a degenerate case of the completely symmetric elliptic integral of the second kind. In particular inequalities involving mean under discussion are obtained. Also, bounds in the mean in question are obtained. Bounding expressions are convex combinations of some quantities depending on variables of the mean.

Mathematics subject classification (2010): 26E60, $26 \mathrm{D} 05$.

Keywords and phrases: Bivariate elliptic means, inequalities, Ky Fan inequalities.

\section{REFERENCES}

[1] G. D. Anderson, M. K. Vamanamurthy, M. Vuorinen, Monotonicity rules in calculus, Amer. Math. Monthly 133 (2006), 805-816.

[2] J. M. Borwein, P. B. Borwein, Pi and the AGM - A Study in Analytic Number Theory and Computational Complexity, Wiley, New York, 1987.

[3] B. C. CARlson, Algorithms involving arithmetic and geometric means, Amer. Math. Monthly 78 (1971), 496-505.

[4] S.-B. Chen, Z.-Y. He, Y.-M. ChU, Y.-Q. Song, X.-J. TAO, Note on certain inequalities for Neuman means, J. Inequal. Appl. 2014 2014:370, 10 pages.

[5] Z.-J. Guo, Y.-M. Chu, Y.-Q. Song, X.-J. TAO, Sharp bounds for Neuman means by harmonic, arithmetic, and contra-harmonic means, Abstr. Appl. Anal. Volume 2014, Article ID914242, 8 pages.

[6] Z.-J. Guo, Y. Zhang, Y.-M. CHu, Y.-Q. Song, Sharp bounds for Neuman means in terms of geometric, arithmetic and quadratic means, arXiv: 1405, 4384v1, May 2014.

[7] Y.-M. LI, B.-Y. Long, Y.-M. CHU, Sharp bounds for the Neuman-Sándor mean in terms of generalized logarithmic mean, J. Math. Inequal. 4, 4 (2012), 567-577.

[8] E. NEUMAN, Inequalities for the Schwab-Borchardt mean and their applications, J. Math. Inequal. 5, 4 (2011), 601-609.

[9] E. NEUMAN, A note on a certain bivariate mean, J. Math. Inequal. 6, 4 (2012), 637-643.

[10] E. Neuman, Sharp inequalities involving Neuman-Sándor and logarithmic means, J. Math. Inequal. 7, 3 (2013), 413-419.

[11] E. Neuman, Inequalities involving certain bivariate means, J. Inequal. Spec. Functions 4, 4 (2013), 12-20.

[12] E. NEUMAN, A one-parameter family of bivariate mean, J. Math. Inequal. 7, 3 (2013), 399-412.

[13] E. Neuman, On some means derived from the Schwab-Borchardt mean, J. Math. Inequal. 8, 1 (2014), 171-183.

[14] E. NeUmAn, On some means derived from the Schwab-Borchardt mean II, J. Math. Inequal. 8, 2 (2014), 361-370.

[15] E. Neuman, On a new bivariate mean, Aequat. Math. 88 (2014), 277-289.

[16] E. Neuman, Inequalities involving generalized trigonometric and hyperbolic functions, J. Math. Inequal. 8, 4 (2014), 725-736.

[17] E. NEuman, Optimal bounds for certain bivariate means, Issues of Analysis. 7 (21), 1 (2014), $35-43$.

[18] E. Neuman, On a new bivariate mean II, Aequat. Math. 89 (2015), 1031-1040.

[19] E. Neuman, J. SÁndor, On the Schwab-Borchardt mean, Math. Pannon. 14, 2 (2003), $253-266$. 
[20] E. Neuman, J. SÁndor, On the Schwab-Borchardt mean II, Math. Pannon. 17, 1 (2006), 49-59.

[21] W.-M. QIAN, Y.-M. ChU, Refinements and bounds for Neuman means in terms of arithmetic and contra-harmonic means, J. Math. Inequal. 9, 3 (2015), 873-881.

[22] W.-M. QIAN, Z.-H. SHAO, Y.-M. CHU, Sharp inequalities involving Neuman means of the second kind and other bivariate means, J. Math. Inequal. 9, 2 (2015), 531-540.

[23] H.-J. SeIFFert, Problem 887, Nieuw. Arch. Wisk. 11 (1993), 176.

[24] H.-J. SEIFFERT, Aufgabe 16, Würzel 29 (1995), 87.

[25] Y. ZhANG, Y.-M. CHU, Y.-L. JiAng, Sharp geometric mean bounds for Neuman mean, Abstr. Appl. Anal., Volume 2014, Article ID 949815, 6 pages.

[26] T.-H. ZhaO, Y.-M. Chu, B.-Y. LiU, Optimal bounds for Neuman-Sándor mean in terms of arithmetic and contra-harmonic means, Abstr. Appl. Anal., Volume 2012, Article ID 302635, 9 pages. 